\title{
MIMO Experiment Design based on Asymptotic Model Order Theory
}

\author{
Cristian R. Rojas, Håkan Hjalmarsson and Roland Hildebrand
}

\begin{abstract}
In this paper we investigate the problem of designing an input signal for a Multi-Input Multi-Output plant to minimize a control-oriented criterion. By employing Ljung's asymptotic (in model order and sample size) covariance formulas, we determine closed form expressions for the optimal input, which provide direct insight into the effect of the principal directions and gains of the open- and closed-loop transfer functions on the kind of experiment to be applied.
\end{abstract}

\section{INTRODUCTION}

The field of experiment design for system identification [23] has been an area of very intensive research, especially during the last years. During the 70 's, the area started by translating some of the results available in the statistical literature [19, 6, 7] from static to linear dynamic systems, by formulating the experiment design problems in the frequency domain $[25,11,30]$. Special emphasis was put on classical design costs, such as the A-, D-, E-, and Loptimality criteria [18], and on the use of results from convex analysis, such as the Carathéodory Theorem [27] to establish that for general convex criteria and constraints, the optimal input can be constructed as a finite sum of sinusoids [11].

During the 80's, Ljung and Yuan, in a series of papers $[24,22,29]$, developed simple expressions for the covariance of the frequency response of the system, which are valid asymptotically in the model order and the sample size. Using these expressions it became possible to derive closed form formulas for the optimal input under several circumstances. Therefore the field moved towards the use of criteria more related to the final purpose of the model [10].

In the 90's, closed-loop experiment design was further explored $[15,8]$. At the end of the decade, researchers in the area started to make use of the recently developed tools in convex optimization [4] (in particular Linear Matrix Inequalities [3]) to solve problems in experiment design using covariance expressions which are non-asymptotic in the model order (but still asymptotic in the sample size) [21, 17, 2]. However, the price paid for the use of such tools is some loss of insight into the general problem of experiment design.

One aspect of experiment design where intuition is greatly required is the one related to Multiple-Input Multiple-Output (MIMO) systems. The problem of designing test signals for MIMO plants has been studied since the beginning of the field [31, 29, 33, 1]. It is well known that for some MIMO

C. R. Rojas and H. Hjalmarsson are with the ACCESS Linnaeus Center, Electrical Engineering, KTH - Royal Institute of Technology, S-100 44 Stockholm, Sweden. Emails: \{cristian.rojas|hakan.hjalmarsson\}@ee.kth.se.

R. Hildebrand is with the Laboratoire Jean Kuntzmann,Université Grenoble 1/CNRS, BP 53, 38041 Grenoble Cedex 9, France. Email: roland.hildebrandeimag.fr. plants, the so-called ill-conditioned ones, identification and control can be very difficult $[20,16]$. This phenomenon can have even worse consequences when subspace identification techniques are employed [26].

In this paper we attempt to obtain some insight into the problem of optimal input design for MIMO systems. To this end, we consider the problem of designing an input signal to improve the accuracy of the model to be used for the design of a controller, according to a predetermined control strategy. Furthermore, to obtain a closed form expression, we recur to the asymptotic (in model order) covariance expressions developed by Yuan and Ljung. The results are then specialized to the case of model reference control, for which we provide some interpretations related to the optimal input.

The paper is structured as follows: Section II introduces the problem and the main assumptions. In Section III we derive first order perturbations for the closed loop expressions when an estimated model is used. Section IV shows the asymptotic (in sample size) covariance for the closed loop output, and in Section V we simplify those expressions by using Yuan and Ljung's theory. From these expressions, we determine in Section VI an input which minimizes the output covariance. Later, in Section VII we specialize the results to the case of model reference control, and we give some interpretations in Section VIII. Section IX presents an illustrative example of a distillation column, and the paper is concluded in Section X.

\section{PROBLEM SETUP}

Consider a linear time invariant system described by

$$
y_{t}=G(q) u_{t}+H(q) w_{t}
$$

where $u_{t} \in \mathbb{R}^{n}$ is the input, $y_{t} \in \mathbb{R}^{n}$ is the output, $G(q)$ and $H(q)$ are rational transfer functions in the forward shift operator $q$, and $w_{t} \in \mathbb{R}^{n}$ is white noise of zero mean and covariance matrix equal to the identity. For simplicity, in the sequel we will omit $q$. The input is to be generated by a feedback mechanism of the form

$$
u_{t}=C\left[r_{t}-y_{t}\right]
$$

where $r_{t} \in \mathbb{R}^{n}$ is a quasi-stationary reference signal [23], independent of $w_{t}$. By combining (1) and (2) we note that the closed loop is described by

$$
y_{t}=(I+G C)^{-1} G C r_{t}+(I+G C)^{-1} H w_{t}
$$

Let us assume that we want to design $C$ using a design method such that at a frequency $\omega, C\left(e^{j \omega}\right)$ depends exclusively on $G\left(e^{j \omega}\right)$ and $H\left(e^{j \omega}\right)$ in a complex differentiable way (c.f. Assumptions A.2 of [15]). 
In practice, $G$ is not exactly known, so in order to design $C$ we need to rely on estimates $\hat{G}$ and $\hat{H}$ of the system transfer functions. We will assume that such estimates are obtained via PEM [23] with an open loop experiment. Using this estimate, the closed loop equation which is actually obtained is

$$
y_{t}=(I+G \hat{C})^{-1} G \hat{C} r_{t}+(I+G \hat{C})^{-1} H w_{t}
$$

where $\hat{C}=C(\hat{G}, \hat{H})$. Our goal is to design an input signal, to be used during the identification step, in order to obtain a model such that $E\left\{\left\|y_{t}-y_{t}^{o}\right\|_{2}^{2}\right\}$ as small as possible.

\section{ASYMPTOTIC EXPRESSIONS FOR THE CLOSED LOOP}

Since PEM estimators are consistent under mild conditions when there is no undermodelling, we can assume that $\hat{G}$ is very close to $G$. Therefore, by writing $\hat{G}=G+\Delta G$, $\hat{H}=H+\Delta H$ and $\hat{C}=C+\Delta C$, and considering only terms of up to first order in $\Delta G, \Delta H$ and $\Delta C$, we have that

$$
\begin{aligned}
(I+G \hat{C})^{-1} & =(I+G C+G \Delta C)^{-1} \\
& =\left(I+(I+G C)^{-1} G \Delta C\right)^{-1}(I+G C)^{-1} \\
& \approx(I-S G \Delta C) S \\
& =S-S G \Delta C S
\end{aligned}
$$

and

$$
\begin{aligned}
(I+G \hat{C})^{-1} G \hat{C} & \approx(I-S G \Delta C) S G(C+\Delta C) \\
& \approx T-S G \Delta C T+S G \Delta C \\
& =T+S G \Delta C S
\end{aligned}
$$

where we have used the first-order approximation $(I+$ $\Delta)^{-1} \approx I-\Delta$, and

$$
\begin{aligned}
& S:=(I+G C)^{-1} \\
& T:=(I+G C)^{-1} G C=I-S
\end{aligned}
$$

Based on (4) and (5), (3) can be approximated as

$$
\begin{aligned}
y_{t} & \approx(T+S G \Delta C S) r_{t}+(S-S G \Delta C S) H w_{t} \\
& =y_{t}^{o}+S G \Delta C S\left[r_{t}-H w_{t}\right]
\end{aligned}
$$

where

$$
y_{t}^{o}:=T r_{t}+S H w_{t}
$$

denotes the output that would be obtained if we designed $C$ based on the true plant.

\section{ASYMPTOTIC COVARIANCE OF THE OUTPUT}

From (6) we have that

$$
E\left\{\left\|y_{t}-y_{t}^{o}\right\|_{2}^{2}\right\} \approx E\left\{\left\|S G \Delta C S\left[r_{t}-H w_{t}\right]\right\|_{2}^{2}\right\}
$$

Notice that the expectation has to be taken with respect to $r_{t}, w_{t}$ and $\Delta G$, which are independent and have zero mean. Therefore,

$$
\begin{aligned}
& E\left\{\left\|y_{t}-y_{t}^{o}\right\|_{2}^{2}\right\} \\
& \approx E\left\{E\left\{\left\|S G \Delta C S\left[r_{t}-H w_{t}\right]\right\|_{2}^{2} \mid \Delta C\right\}\right\} \\
& \approx E\left\{\frac{1}{2 \pi} \int S G \Delta C S\left[\Phi_{r}+\Phi_{v}\right] S^{H} \Delta C^{H} G^{H} S^{H}\right\}
\end{aligned}
$$

where $\Phi_{v}:=H H^{H}$, and we have used Parseval's relation. Here and in the sequel we have omitted the arguments $e^{j \omega}$ and the limits of integration (from $-\pi$ to $\pi$ ) for simplicity. This expression can be further simplified by factorizing $\Phi_{r}+$ $\Phi_{v}$ as $R R^{H}$ (only as an intermediate step) and using the vec operator [5]:

$$
\begin{gathered}
E\left\{\frac{1}{2 \pi} \int S G \Delta C S\left[\Phi_{r}+\Phi_{v}\right] S^{H} \Delta C^{H} G^{H} S^{H}\right\} \\
=E\left\{\frac{1}{2 \pi} \int S G \Delta C S R R^{H} S^{H} \Delta C^{H} G^{H} S^{H}\right\} \\
=E\left\{\frac{1}{2 \pi} \int\|\operatorname{vec}\{S G \Delta C S R\}\|_{2}^{2}\right\}
\end{gathered}
$$

Now,

$$
\operatorname{vec}\{S G \Delta C S R\}=\left[R^{T} S^{T} \otimes S G\right] \operatorname{vec}\{\Delta C\}
$$

where $\otimes$ denotes the Kronecker product. Therefore, (7) can be written as

$$
\begin{aligned}
E & \left\{\frac{1}{2 \pi} \int\|\operatorname{vec}\{S G \Delta C S R\}\|_{2}^{2}\right\} \\
& =E\left\{\frac{1}{2 \pi} \int\left\|\left[R^{T} S^{T} \otimes S G\right] \operatorname{vec}\{\Delta C\}\right\|_{2}^{2}\right\} \\
& =\frac{1}{2 \pi} \int \operatorname{tr}\left[\left(S^{*} R^{*} R^{T} S^{T} \otimes G^{H} S^{H} S G\right) P_{C}\right] \\
& =\frac{1}{2 \pi} \int \operatorname{tr}\left\{\left(S^{*}\left[\Phi_{r}^{*}+\Phi_{v}^{*}\right] S^{T} \otimes G^{H} S^{H} S G\right) P_{C}\right\}
\end{aligned}
$$

where

$$
P_{C}:=\operatorname{Cov}\{\operatorname{vec}[\hat{C}]\}
$$

To continue, it is necessary to obtain an expression for $P_{C}$. To this end, notice that the assumptions considered in Section II imply that

$$
\begin{aligned}
\Delta C & \approx \frac{\partial \operatorname{vec} C}{\partial \operatorname{vec} G} \Delta G+\frac{\partial \operatorname{vec} C}{\partial \operatorname{vec} H} \Delta H \\
& =\left[\begin{array}{ll}
\frac{\partial \operatorname{vec} C}{\partial \operatorname{vec} G} & \frac{\partial \operatorname{vec} C}{\partial \operatorname{vec} H}
\end{array}\right] \operatorname{vec}\left[\begin{array}{ll}
\Delta G & \Delta H
\end{array}\right]
\end{aligned}
$$

Hence, $P_{C}$ is given by

$$
P_{C}=\left[\begin{array}{ll}
\frac{\partial \operatorname{vec} C}{\partial \operatorname{vec} G} & \frac{\partial \operatorname{vec} C}{\partial \operatorname{vec} H}
\end{array}\right] P_{[\hat{G} \hat{H}]}\left[\begin{array}{ll}
\frac{\partial \operatorname{vec} C}{\partial \operatorname{vec} G} & \frac{\partial \operatorname{vec} C}{\partial \operatorname{vec} H}
\end{array}\right]^{H}
$$

\section{YUAN AND LJUNG'S ASYMPTOTIC COVARIANCE EXPRESSION}

In [29, 32] it was shown that for models with a given shift structures it holds, under mild conditions, that

$$
\lim _{n \rightarrow \infty} \lim _{N \rightarrow \infty} \frac{N}{n} P_{[\hat{G} \hat{H}]}=\left[\begin{array}{cc}
\left(\Phi_{u}^{i d}\right)^{-T} & 0 \\
0 & I
\end{array}\right] \otimes \Phi_{v}
$$

under open loop identification, where $\Phi_{u}^{i d}$ is the spectrum of the input signal used during the identification stage to obtain $\hat{G}$. Equation (10) gives rise to the approximation

$$
P_{[\hat{G} \hat{H}]} \approx \frac{n}{N}\left[\begin{array}{cc}
\left(\Phi_{u}^{i d}\right)^{-T} & 0 \\
0 & I
\end{array}\right] \otimes \Phi_{v}
$$


which holds for models of high order. Introducing (11) into (9) gives

$$
\begin{array}{r}
P_{C} \approx \frac{n}{N}\left\{\frac{\partial \operatorname{vec} C}{\partial \operatorname{vec} G}\left[\left(\Phi_{u}^{i d}\right)^{-T} \otimes \Phi_{v}\right]\left(\frac{\partial \operatorname{vec} C}{\partial \operatorname{vec} G}\right)^{H}\right. \\
\left.+\frac{\partial \operatorname{vec} C}{\partial \operatorname{vec} H}\left[I \otimes \Phi_{v}\right]\left(\frac{\partial \operatorname{vec} C}{\partial \operatorname{vec} H}\right)^{H}\right\}
\end{array}
$$

This implies that (8) can be written as

$$
\begin{aligned}
& E\left\{\left\|y_{t}-y_{t}^{o}\right\|_{2}^{2}\right\} \\
& \approx \frac{n}{2 \pi N} \int \operatorname{tr}\left\{\left(S^{*}\left[\Phi_{r}^{*}+\Phi_{v}^{*}\right] S^{T} \otimes G^{H} S^{H} S G\right) .\right. \\
& \left.\left[\frac{\partial \operatorname{vec} C}{\partial \operatorname{vec} G}\left[\left(\Phi_{u}^{i d}\right)^{-T} \otimes \Phi_{v}\right]\left(\frac{\partial \operatorname{vec} C}{\partial \operatorname{vec} G}\right)^{H}\right]\right\}+J_{o} \\
& =\int \operatorname{tr}\left\{F\left[\left(\Phi_{u}^{i d}\right)^{-T} \otimes \Phi_{v}\right]\right\}+J_{o}
\end{aligned}
$$

where

$$
\begin{aligned}
J_{o}:=\frac{n}{2 \pi N} \int \operatorname{tr}\{ & \left(S^{*}\left[\Phi_{r}^{*}+\Phi_{v}^{*}\right] S^{T} \otimes G^{H} S^{H} S G\right) . \\
& {\left.\left[\frac{\partial \operatorname{vec} C}{\partial \operatorname{vec} H}\left[I \otimes \Phi_{v}\right]\left(\frac{\partial \operatorname{vec} C}{\partial \operatorname{vec} H}\right)^{H}\right]\right\} }
\end{aligned}
$$

is a term which does not depend on $\Phi_{u}^{i d}$, and

$$
\begin{array}{r}
F:=\frac{n}{2 \pi N}\left[\frac{\partial \operatorname{vec} C}{\partial \operatorname{vec} G}\right]^{H}\left(S^{*}\left[\Phi_{r}^{*}+\Phi_{v}^{*}\right] S^{T} \otimes G^{H} S^{H} S G\right) \\
\cdot\left[\frac{\partial \operatorname{vec} C}{\partial \operatorname{vec} G}\right]
\end{array}
$$

\section{OPTIMAL INPUT DESIGN}

From (12) it turns out that the problem of designing $\Phi_{u}^{i d}$ to minimize $E\left\{\left\|y_{t}-y_{t}^{o}\right\|_{2}^{2}\right\}$ subject to a total input power constraint is equivalent to

$$
\begin{array}{ll}
\min _{\Phi_{u}^{i d}} & \int \operatorname{tr}\left\{F\left[\left(\Phi_{u}^{i d}\right)^{-T} \otimes \Phi_{v}\right]\right\} \\
\text { s.t. } & \Phi_{u}^{i d} \geq 0, \\
& \frac{1}{2 \pi} \int \operatorname{tr}\left[\Phi_{u}^{i d}\right] \leq 1
\end{array}
$$

Such an input power constraint does not seem quite realistic, as in practice there are usually power constraints for each component of the input, i.e., for each $\left[\Phi_{u}^{i d}\right]_{i, i}$. However, this constraint might be useful in situations where there are output power constraints, which must be translated into input power constraints, but where the true system is essentially unknown (so the resulting input constraint must consider a worst case scenario). Moreover, the constraint considered here gives rise to a closed form expression for the optimal $\Phi_{u}^{i d}$. This will be seen now.

To solve (14), we can use some tools from calculus of variations [9]. By Lemma 1 of the Appendix, we have that

$$
\frac{\partial}{\partial \Phi_{u}^{i d}} \operatorname{tr}\left\{F\left[\left(\Phi_{u}^{i d}\right)^{-T} \otimes \Phi_{v}\right]\right\}=-\left[\Phi_{u}^{i d}\right]^{-T} Z\left[\Phi_{u}^{i d}\right]^{-T}
$$

and

$$
\frac{\partial}{\partial \Phi_{u}^{i d}} \operatorname{tr}\left\{\Phi_{u}^{i d}\right\}=I
$$

where $Z \in \mathbb{C}^{n \times n}$ is given by

$$
Z_{k l}=\operatorname{tr}\left\{F_{k l} \Phi_{v}\right\}, \quad k, l=1, \ldots, n
$$

and $F_{k l}$ is the $k l$-th $(n \times n)$ block of $F$.

From (15) and (16) we deduce that the optimal input $\Phi_{u}^{i d}$ according to (14) satisfies

$$
\left[\Phi_{u}^{i d}\right]^{-T} Z\left[\Phi_{u}^{i d}\right]^{-T}=\lambda I
$$

for some $\lambda \geq 0$. This gives

$$
\left[\Phi_{u}^{i d}\right]^{\text {opt }} \propto Z^{T / 2}
$$

\section{MODEL REFERENCE CONTROL}

As an example of the results obtained in the previous sections, we consider next the problem of model reference control. This means that the control design method consists in choosing $C$ such that the transfer function from $r_{t}$ to $y_{t}$ is equal to a given transfer function $T$, i.e.,

$$
T=(I+G C)^{-1} G C
$$

Solving for $C$ in (18) gives

$$
C=G^{-1} T(I-T)^{-1}
$$

To proceed, we need an expression for $\partial(\operatorname{vec} C) / \partial(\operatorname{vec} G)$. This can be obtained as follows:

$$
\begin{aligned}
\hat{C} & =\hat{G}^{-1} T(I-T)^{-1} \\
& =(G+\Delta G)^{-1} T(I-T)^{-1} \\
& =\left(I+G^{-1} \Delta G\right)^{-1} G^{-1} T(I-T)^{-1} \\
& \approx\left(I-G^{-1} \Delta G\right) G^{-1} T(I-T)^{-1} \\
& =C-G^{-1} \Delta G C
\end{aligned}
$$

Hence,

$$
\Delta C \approx-G^{-1} \Delta G C
$$

or

$$
\operatorname{vec}\{\Delta C\} \approx-\left[C^{T} \otimes G^{-1}\right] \operatorname{vec}\{\Delta G\}
$$

Therefore,

$$
\frac{\partial \operatorname{vec} C}{\partial \operatorname{vec} G}=-C^{T} \otimes G^{-1}
$$

Introducing this expression into (13) gives

$$
\begin{gathered}
F=\frac{n}{2 \pi N}\left(C^{*} \otimes G^{-H}\right)\left(S^{*}\left[\Phi_{r}^{*}+\Phi_{v}^{*}\right] S^{T} \otimes G^{H} S^{H} S G\right) \\
\cdot\left(C^{T} \otimes G^{-1}\right) \\
=\frac{n}{2 \pi N} C^{*} S^{*}\left[\Phi_{r}^{*}+\Phi_{v}^{*}\right] S^{T} C^{T} \otimes S^{H} S
\end{gathered}
$$

Hence, the expression for $Z$ (c.f. (17)) is

$$
\begin{aligned}
Z_{k l} & =\frac{n}{2 \pi N} \operatorname{tr}\left\{\left(C^{*} S^{*}\left[\Phi_{r}^{*}+\Phi_{v}^{*}\right] S^{T} C^{T}\right)_{k l} S^{H} S \Phi_{v}\right\} \\
& =\frac{n}{2 \pi N}\left(C^{*} S^{*}\left[\Phi_{r}^{*}+\Phi_{v}^{*}\right] S^{T} C^{T}\right)_{k l} \operatorname{tr}\left\{S \Phi_{v} S^{H}\right\}
\end{aligned}
$$


for $k, l=1, \ldots, n$, or

$$
Z=\frac{n}{2 \pi N} \operatorname{tr}\left\{S \Phi_{v} S^{H}\right\} C^{*} S^{*}\left[\Phi_{r}^{*}+\Phi_{v}^{*}\right] S^{T} C^{T}
$$

This implies that the optimal input is given by

$$
\begin{aligned}
{\left[\Phi_{u}^{i d}\right]^{o p t} } & \propto Z^{T / 2} \\
& \propto \sqrt{\operatorname{tr}\left\{S \Phi_{v} S^{H}\right\}}\left[C S\left[\Phi_{r}+\Phi_{v}\right] S^{H} C^{H}\right]^{1 / 2}
\end{aligned}
$$

\section{INTERPRETATIONS}

The expression (19) for the optimal input involves two factors. The first one corresponds to the square root of the power of the noise as seen from the output, i.e., the power of $S(q) H(q) w_{t}$. For those frequencies where this noise is "small", the input signal does not have to be very powerful in order to obtain a reasonable model for control. This can be due to two reasons:

1) $S$ is small at those frequencies, which means that $T \approx 1$, or equivalently, $C$ has very high gain at those frequencies. This implies that modeling errors can be reasonably tolerated, as it is well known from the "forgiving nature of feedback" [13].

2) $H$ is small at those frequencies. In this case, the effect of disturbances is low, which allows us to obtain a reasonably good model without much input power.

As for the second factor in (19), we may notice that $C S\left[\Phi_{r}+\right.$ $\left.\Phi_{v}\right] S^{H} C^{H}$ corresponds to the spectrum of the input which would be applied, in principle, to the system during closed loop operation. This expression can be factorized as

$$
C S\left[\Phi_{r}+\Phi_{v}\right] S^{H} C^{H}=U U^{H}
$$

Thus, (19) can be written as

$$
\left[\Phi_{u}^{i d}\right]^{o p t} \propto \sqrt{\operatorname{tr}\left\{S \Phi_{v} S^{H}\right\}}\left[U U^{H}\right]^{1 / 2}
$$

Therefore, we can conclude that (the square of) the optimal input to be applied should be essentially the same as the input which will be effectively applied to the plant during the closed loop operation, weighted by the total power spectral density of the noise.

Notice however, that the principal directions (and the relative principal gains) of the input are completely determined by those of the input to be applied during closed loop operation. Thus, the optimal input tries to recreate the normal operating conditions. This has already been noticed before, e.g., in identification for minimum variance control [10, 15, 12], and developed further in [14].

\section{ILLUSTRATIVE EXAMPLE}

To illustrate the results of Section VII, consider an illconditioned simple $2 \times 2$ model of a distillation column [28, Section 3.7.2]:

$$
G(s)=\frac{1}{75 s+1}\left[\begin{array}{cc}
87.8 & -86.4 \\
108.2 & -109.6
\end{array}\right]
$$

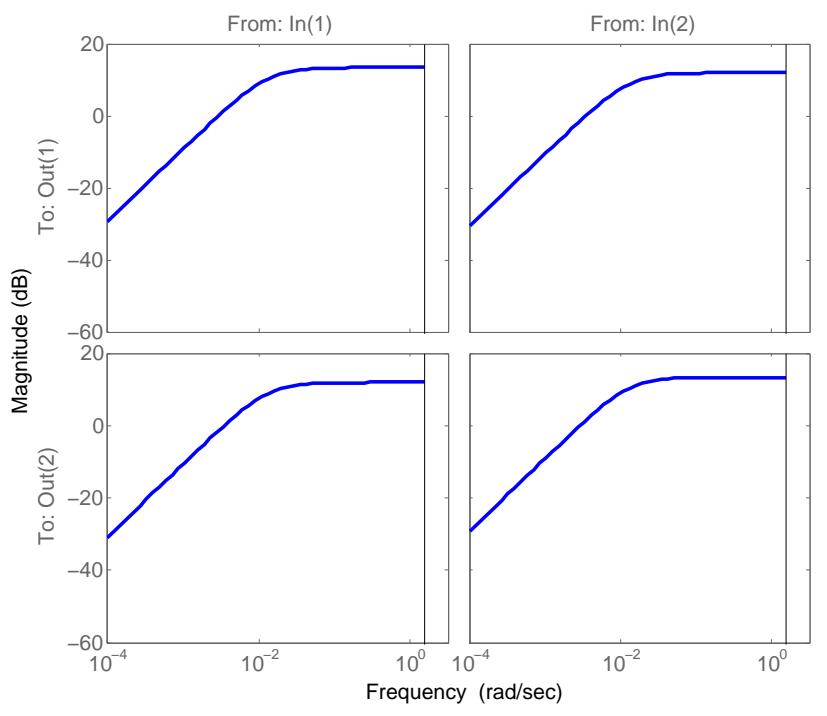

Fig. 1. Optimal input spectrum for the distillation column of Section IX.

where $s$ is the Laplace variable, and time is measured in minutes. The model is sampled every 2 minutes with a zeroorder hold, thus obtaining the equivalent discrete-time model:

$$
G_{d}(q)=\frac{1}{q-0.9737}\left[\begin{array}{cc}
0.0231 & -0.02274 \\
0.02847 & -0.02884
\end{array}\right]
$$

For simplicity, let us assume that the disturbance model is of the form

$$
H_{d}(q)=\frac{1}{q-0.9737}\left[\begin{array}{cc}
0.00001 & 0 \\
0 & 0.00001
\end{array}\right]
$$

We want to design a controller so that the transfer function from $r$ to $y$ in continuous time is equal to

$$
T(s)=\frac{1}{75 s+1} I
$$

To this end, we estimate via Least Squares [23] a model of the plant of the form

$$
G_{d}(q)=\frac{1}{z-a}\left[\begin{array}{ll}
b_{11} & b_{12} \\
b_{21} & b_{22}
\end{array}\right] ; \quad H_{d}(q)=\frac{\lambda}{z-a} I
$$

The optimal input spectrum, according to (19), is

$$
\begin{array}{r}
{\left[\Phi_{u}^{i d}\right]^{\text {opt }} \propto \sqrt{\frac{z-2+z^{-1}}{-0.97369 z+1.94806-0.97369 z^{-1}}}} \\
\cdot\left[\begin{array}{ll}
50.86022 & 50.81780 \\
50.81780 & 50.78046
\end{array}\right]
\end{array}
$$

and its shape is shown in Figure 1. Notice that the input is almost singular, since it tries to invert the plant dynamics by recreating the closed loop operation. Since the spectrum is non-rational, it is approximated by a Padé-approximant of order 4 (for simulation purposes).

To examine the performance of the controller designed with a estimated model based on the optimal input, 1000 Monte Carlo simulations are performed, each using 10000 samples for identification and taking 10000 samples to estimate the performance index $E\left\{\left\|y_{t}-y_{t}^{o}\right\|_{2}^{2}\right\}$ as an average. 
TABLE I

ESTIMATED NORMALIZED PERFORMANCE FOR THE CONTROLLERS BASED ON WHITE NOISE AND THE OPTIMAL INPUT

\begin{tabular}{|c|c|c|}
\hline & White Noise & Optimal Input \\
\hline Average Performance & 0.00288 & 0.00226 \\
Standard Deviation & 0.00146 & 0.00099 \\
\hline
\end{tabular}

For comparison purposes, the result is contrasted with the use of white noise of unit covariance matrix for the identification of the model. The results (normalized by multiplying them with the number of samples) are shown in Table I. From these results we see an improvement of $21.6 \%$ in using an optimal input compared to white noise.

\section{CONCLUSIONS}

In this paper we have studied the problem of optimal input design for MIMO systems, when the final purpose of the model is to design a controller using a predetermined strategy. Since our purpose has been to obtain some insight on the role of the optimal input, asymptotic (in model order and sample size) covariance expressions have been used. In the particular case of model reference control, we have seen that the optimal input tries to recreate the closed loop operation, i.e., it attempts to invert the plant in order to obtain the designed closed loop transfer function. This effect has been illustrated with an example, where it has been shown that the performance improved when using the optimally designed input.

\section{APPENDIX}

Lemma 1: Let $M \in \mathbb{C}^{m \times m} ; B \in \mathbb{C}^{p \times p}$ be invertible; and $Q \in \mathbb{C}^{m p \times m p}$ be a block matrix with blocks $Q_{k l} \in \mathbb{C}^{m \times m}$ $(k, l=1, \ldots, p)$. Then,

$$
\frac{\partial}{\partial B} \operatorname{tr}\left\{Q\left(B^{-T} \otimes M\right)\right\}=-B^{-T} Z B^{-T}
$$

where $Z \in \mathbb{C}^{p \times p}$ is given by $Z_{k l}=\operatorname{tr}\left\{Q_{k l} M\right\}$.

Proof: The proof is very similar to that of $[29$, Lemma 2.1]. Since

$$
B^{-T} B^{T}=I
$$

we have

$$
\frac{\partial B^{-T}}{\partial B_{i j}} B^{T}+B^{-T} \frac{\partial B^{T}}{\partial B_{i j}}=0
$$

Therefore,

$$
\frac{\partial B^{-T}}{\partial B_{i, j}}=-B^{-T} \frac{\partial B^{T}}{\partial B_{i j}} B^{-T}=-B^{-T} e_{j} e_{i}^{T} B^{-T}
$$

where $e_{i}$ is the $i$-th unit vector. Therefore, with the definition of $Z$ given above,

$$
\begin{aligned}
\frac{\partial}{\partial B_{i j}} \operatorname{tr}\{Q & \left.\left(B^{-T} \otimes M\right)\right\} \\
= & \operatorname{tr}\left\{Q\left(\frac{\partial B^{-T}}{\partial B_{i j}} \otimes M\right)\right\} \\
= & -\operatorname{tr}\left\{Q\left(B^{-T} e_{j} e_{i}^{T} B^{-T} \otimes M\right)\right\} \\
& =-\sum_{k, l=1}^{p} \operatorname{tr}\left\{Q_{k l}\left(B^{-T} e_{j} e_{i}^{T} B^{-T}\right)_{l k} M\right\} \\
& =-\sum_{k, l=1}^{p}\left(B^{-T} e_{j} e_{i}^{T} B^{-T}\right)_{l k} \operatorname{tr}\left\{Q_{k l} M\right\} \\
& =-\operatorname{tr}\left\{B^{-T} e_{j} e_{i}^{T} B^{-T} Z\right\} \\
& =\left[-B^{-T} Z B^{-T}\right]_{i j}
\end{aligned}
$$

This concludes the proof.

\section{REFERENCES}

[1] Barenthin, M., Bombois, X., Hjalmarsson, H. and Scorletti, G. [2008]. Identification for control of multivariable systems: Controller validation and experiment design via LMIs, Automatica 44(12): 3070-3078.

[2] Bombois, X., Scorletti, G., Gevers, M., Van den Hof, P. and Hildebrand, R. [2006]. Least costly identification experiment for control, Automatica 42(10): 1651-1662.

[3] Boyd, S., El Ghaoui, L., Feron, E. and Balakrishnan, V. [1994]. Linear Matrix Inequalities in System and Control Theory, SIAM Studies in Applied Mathematics, Philadelphia, USA.

[4] Boyd, S. and Vandenberghe, L. [2003]. Convex Optimization, Cambridge University Press.

[5] Brewer, J. W. [1978]. Kronecker products and matrix calculus in system theory, IEEE Transactions on Circuits and Systems 25(9): 772-781.

[6] Cox, D. R. [1958]. Planning of Experiments, Wiley, New York.

[7] Fedorov, V. V. [1972]. Theory of Optimal Experiments, Academic Press, New York.

[8] Forssell, U. and Ljung, L. [2000]. Some results on optimal experiment design, Automatica 36(5): 749-756.

[9] Gelfand, I. M. and Fomin, S. V. [1963]. Calculus of Variations, Dover, Mineola, New York.

[10] Gevers, M. and Ljung, L. [1986]. Optimal experiment designs with respect to the intended model application, Automatica 22(5): 543-554.

[11] Goodwin, G. C. and Payne, R. L. [1977]. Dynamic System Identification: Experiment Design and Data Analysis, Academic Press, New York.

[12] Hildebrand, R. and Solari, G. [2005]. Identification for control: optimal input intended to identify a minimum variance controller, Automatica 43: 758-767.

[13] Hjalmarsson, H. [2005]. From experiment design to closed-loop control, Automatica 41(3): 393-438.

[14] Hjalmarsson, H. [2009]. System identification of 
complex and structured systems, Proceedings of the European Control Conference, Budapest, Hungary.

[15] Hjalmarsson, H., Gevers, M. and De Bruyne, F. [1996]. For model-based control design, closed loop identification gives better performance, Automatica 32(12): 1659-1673.

[16] Jacobsen, E. W. and Skogestad, S. [1994]. Inconsistencies in dynamic models for ill-conditioned plants: application to low-order models of distillation columns, Industry and Engineering Chemistry Research 33: 631640.

[17] Jansson, H. and Hjalmarsson, H. [2005]. Input design via LMIs admitting frequency-wise model specifications in confidence regions, IEEE Transactions on Automatic Control 50(10): 1534-1549.

[18] Kiefer, J. [1974]. General equivalence theory for optimum designs (aproximate theory), Annals of Statistics 2(5): 849-879.

[19] Kiefer, J. and Wolfowitz, J. [1960]. The equivalence of two extremum problems, Canadian Journal of Mathematics 12: 363-366.

[20] Koung, C.-W. and MacGregor, J. F. [1993]. Design of identification experiments for robust control. a geometric approach for bivariate processes, Industry and Engineering Chemistry Research 32: 1658-1666.

[21] Lindqvist, K. and Hjalmarsson, H. [2000]. Optimal input design using linear matrix inequalities, IFAC Symposium on System Identification.

[22] Ljung, L. [1985]. Asymptotic variance expressions for identified black-box transfer function models, IEEE Transactions on Automatic Control 30(9): 834-844.

[23] Ljung, L. [1999]. System Identification: Theory for the User, 2nd Edition, Prentice Hall, Upper Saddle River, New Jersey.

[24] Ljung, L. and Yuan, Z.-D. [1985]. Asymptotic properties of black-box identification of transfer functions, IEEE Transactions on Automatic Control AC-30: 514530.

[25] Mehra, R. K. [1974]. Optimal input signals for parameter estimation in dynamic systems - survey and new results, IEEE Transactions on Automatic Control 19(6): 753-768.

[26] Micchi, A. and Pannocchia, G. [2008]. Comparison of input signals in subspace identification of multivariable ill-conditioned systems, Journal of Process Control 18: 582-593.

[27] Rockafellar, R. T. [1970]. Convex Analysis, Princeton University Press, Princeton, New Jersey.

[28] Skogestad, S. and Postlethwaite, I. [2005]. Multivariable Feedback Control - Analysis and Design, 2nd Edition, John Wiley \& Sons, West Sussex, England.

[29] Yuan, Z. D. and Ljung, L. [1984]. Black-box identification of multivariable transfer functions - asymptotic properties and optimal input design, International Journal of Control 40(2): 233-256.

[30] Zarrop, M. [1979]. Optimal Experiment Design for Dynamic System Identification, Springer, Berlin.
[31] Zarrop, M. B., Payne, R. L. and Goodwin, G. C. [1979]. Experiment design for time series analysis: the multivariate case, SIAM Journal on Applied Mathematics 37(2): 370-381.

[32] Zhu, Y. [1989]. Black-box identification of MIMO transfer functions: asymptotic properties of prediction error models, International Journal of Adaptive Control and Signal Processing 3: 357-373.

[33] Zhu, Y. C. [1998]. Multivariable process identification for MPC: the asymptotic method and its applications, Journal of Process Control 8(2): 101-115. 